\title{
GENERALIZED JORDAN TRIPLE HIGHER DERIVATIONS ON SEMIPRIME RINGS
}

\author{
Feng Wei and Zhankui Xiao
}

\begin{abstract}
In this paper we prove that every generalized Jordan triple higher derivation on a 2-torsion free semiprime ring is a generalized higher derivation. This extend the main result of $[9]$ to the case of a semiprime ring.
\end{abstract}

\section{Introduction and preliminaries}

Let $\mathcal{R}$ be an associative ring not necessarily with identity element. A derivation (resp. Jordan derivation) of $\mathcal{R}$ is an additive map $d: \mathcal{R} \longrightarrow \mathcal{R}$ such that $d(x y)=d(x) y+x d(y)$ for all $x, y \in \mathcal{R}$ (resp. $d\left(x^{2}\right)=d(x) x+x d(x)$ for all $x \in \mathcal{R}$ ). Obviously, every derivation is a Jordan derivation. The converse is in general not true. Herstein [6] proved that the converse is true on a 2-torsion free prime ring. Subsequently, Cusack and Brešar independently extended this result to the case of a 2-torsion free semiprime ring in [4] and [2], respectively. The following two definitions are corresponding to derivations and Jordan derivations, respectively. One is a common extension of derivation, and another is a generalization of Jordan derivation. An additive map $\mu: \mathcal{R} \longrightarrow \mathcal{R}$ is called a generalized derivation of $\mathcal{R}$ if there exists a derivation $d$ of $\mathcal{R}$ such that

$$
\mu(x y)=\mu(x) y+x d(y)
$$

for all $x, y \in \mathcal{R}$. $d$ is called an associated derivation of $\mu$. An additive map $\mu: \mathcal{R} \longrightarrow \mathcal{R}$ is called a generalized Jordan derivation of $\mathcal{R}$ if there exists a Jordan derivation $d$ of $\mathcal{R}$ such that

$$
\mu\left(x^{2}\right)=\mu(x) x+x d(x)
$$

for all $x \in \mathcal{R}$. The map $d$ is called an associated Jordan derivation of $\mu$. When $\mathcal{R}$ is a 2 -torsion free ring, this definition is equivalent to saying that there exists

Received July 10, 2008; Revised September 12, 2008.

2000 Mathematics Subject Classification. 16W25, 16N60.

Key words and phrases. generalized Jordan triple higher derivation, semi-prime ring.

This work is partially supported by the National Natural Science Foundation of China (Grant No. 10871023). 
a Jordan derivation $d$ of $\mathcal{R}$ such that

$$
\mu(x y+y x)=\mu(x) y+x d(y)+\mu(y) x+y d(x)
$$

for all $x, y \in \mathcal{R}$. Likewise, every generalized derivation is a generalized Jordan derivation. This converse is also in general false. Argac and Albas [1] gave a counterexample with respect to this. But, Jing and Lu proved that every generalized Jordan derivation on a 2-torsion free prime ring is a generalized derivation indeed. The authors [13] extended this conclusion to the case of a semiprime ring. An additive map $f: \mathcal{R} \longrightarrow \mathcal{R}$ is called a Jordan triple derivation of $\mathcal{R}$ if

$$
f(x y x)=f(x) y x+x f(y) x+x y f(x)
$$

for all $x, y \in \mathcal{R}$. Brešar proved that every Jordan triple derivation on a 2torsion free semiprime ring is a derivation. It turns out that every Jordan derivation on a 2-torsion free ring is a Jordan triple derivation [3]. One natural generalization of Jordan triple derivation is so called generalized Jordan triple derivation. An additive map $\delta: \mathcal{R} \longrightarrow \mathcal{R}$ is called a generalized Jordan triple derivation of $\mathcal{R}$ if there exists a Jordan triple derivation $f$ of $\mathcal{R}$ such that

$$
\delta(x y x)=\delta(x) y x+x f(y) x+x y f(x)
$$

for all $x, y \in \mathcal{R}$. The map $f$ is called an associated Jordan triple derivation of $\delta$. Jing and Lu proved that every generalized Jordan triple derivation on a 2 -torsion free prime ring is a generalized derivation [8]. The authors [13] proved that this result remains valid for a semiprime ring.

On the other hand, many different kinds of higher derivations, which are consisting of family of some additive maps, also have been considered in commutative and noncommutative rings. Let $\mathbb{N}_{0}$ be the set of all nonnegative integers and let $D=\left(d_{i}\right)_{i \in \mathbb{N}_{0}}$ be a family of additive maps on a ring $\mathcal{R}$ such that $d_{0}=i d_{\mathcal{R}} . D$ is called to be:

(a) a higher derivation if for each $n \in \mathbb{N}_{0}$,

$$
d_{n}(x y)=\sum_{i+j=n} d_{i}(x) d_{j}(y)
$$

for all $x, y \in \mathcal{R}$;

(b) a Jordan higher derivation if for each $n \in \mathbb{N}_{0}$,

$$
d_{n}\left(x^{2}\right)=\sum_{i+j=n} d_{i}(x) d_{j}(x)
$$

for all $x \in \mathcal{R}$;

(c) a Jordan triple higher derivation if for each $n \in \mathbb{N}_{0}$,

$$
d_{n}(x y x)=\sum_{i+j+k=n} d_{i}(x) d_{j}(y) d_{k}(x)
$$

for all $x, y \in \mathcal{R}$. 
Let $\mathbb{N}_{0}$ be the set of all nonnegative integers and let $\Delta=\left(\delta_{i}\right)_{i \in \mathbb{N}_{0}}$ be a family of additive maps on a ring $\mathcal{R}$ such that $\delta_{0}=i d_{\mathcal{R}} . \Delta$ is said to be:

(a) a generalized higher derivation if there exists a higher derivation $D=$ $\left(d_{i}\right)_{i \in \mathbb{N}_{0}}$ such that

$$
\delta_{n}(x y)=\sum_{i+j=n} \delta_{i}(x) d_{j}(y)
$$

for all $x, y \in \mathcal{R}$ and for each $n \in \mathbb{N}_{0}$. D is called an associated higher derivation of $\Delta$;

(b) a generalized Jordan higher derivation if there exists a Jordan higher derivation $D=\left(d_{i}\right)_{i \in \mathbb{N}_{0}}$ such that

$$
\delta_{n}\left(x^{2}\right)=\sum_{i+j=n} \delta_{i}(x) d_{j}(x)
$$

for all $x \in \mathcal{R}$ and for each $n \in \mathbb{N}_{0}$. D is called an associated Jordan higher derivation of $\Delta$;

(c) a generalized Jordan triple higher derivation if there exists a Jordan triple higher derivation $D=\left(d_{i}\right)_{i \in \mathbb{N}_{0}}$ such that

$$
\delta_{n}(x y x)=\sum_{i+j+k=n} \delta_{i}(x) d_{j}(y) d_{k}(x)
$$

for all $x, y \in \mathcal{R}$ and for each $n \in \mathbb{N}_{0}$. D is called an associated Jordan triple higher derivation of $\Delta$.

In [5], Ferrero and Haetinger obtained the following conclusion. Let $\mathcal{R}$ be a semiprime ring of characteristic not 2 (resp. prime ring of characteristic not 2 and $\mathcal{L}$ be an admissible Lie ideal of $\mathcal{R}$ ). Then every Jordan triple higher derivation of $\mathcal{R}$ (resp. of $\mathcal{L}$ into $\mathcal{R}$ ) is a higher derivation of $\mathcal{R}$ (resp. of $\mathcal{L}$ into $\mathcal{R}$ ). Recently, Jung proved that every generalized Jordan triple higher derivation on a 2-torsion free prime ring is a generalized higher derivation [9].

The main objective of this paper is to prove that every generalized Jordan triple higher derivation on a 2-torsion free semiprime ring is a generalized higher derivation, which extends the main theorem of [9] to the case of a semiprime ring.

\section{Generalized Jordan triple higher derivations on semiprime rings}

Throughout this paper $\mathcal{R}$ always denotes an associative ring with the center $\mathcal{C}_{\mathcal{R}}$. $\Delta$ always denotes a generalized Jordan triple higher derivation on $\mathcal{R}$ with the associated Jordan triple higher derivation $D$. A ring $\mathcal{R}$ is said to be $n$ torsion free if $n x=0$ implies $x=0$ for all $x \in \mathcal{R}$. As usual, we denote the commutator $x y-y x$ by $[x, y]$. For any $x, y, z \in \mathcal{R}$, we set $Q(x, y, z)=x y z-z y x$. Let $\Delta=\left(\delta_{i}\right)_{i \in \mathbb{N}_{0}}$ be a generalized Jordan triple higher derivation on $\mathcal{R}$ with the associated Jordan triple higher derivation $D=\left(d_{i}\right)_{i \in \mathbb{N}_{0}}$. For all $x, y, z \in \mathcal{R}$ 
and each $n \in \mathbb{N}_{0}$, we write $P_{n}(x, y, z)=\delta_{n}(x y z)-\sum_{i+j+k=n} \delta_{i}(x) d_{j}(y) d_{k}(z)$. We should remark that

$$
\delta_{n}(x y z+z y x)=\sum_{i+j+k=n} \delta_{i}(x) d_{j}(y) d_{k}(z)+\sum_{i+j+k=n} \delta_{i}(z) d_{j}(y) d_{k}(x)
$$

for all $x, y, z \in \mathcal{R}$ and each $n \in \mathbb{N}_{0}$.

Before proving the main theorem, we must recall some basic facts.

Lemma 2.1 ([3, Lemma 1.2]). Let $\mathcal{G}_{1}, \mathcal{G}_{2}, \ldots, \mathcal{G}_{n}$ be additive groups and $\mathcal{R}$ be a semiprime ring. Suppose that maps $S: \mathcal{G}_{1} \times \mathcal{G}_{2} \times \cdots \times \mathcal{G}_{n} \longrightarrow \mathcal{R}$ and $T: \mathcal{G}_{1} \times \mathcal{G}_{2} \times \cdots \times \mathcal{G}_{n} \longrightarrow \mathcal{R}$ are both additive in each variable. If

$$
S\left(a_{1}, a_{2}, \ldots, a_{n}\right) r T\left(a_{1}, a_{2}, \ldots, a_{n}\right)=0
$$

for all $r \in \mathcal{R}, a_{i} \in \mathcal{G}_{i}, i=1,2, \ldots, n$, then

$$
S\left(a_{1}, a_{2}, \ldots, a_{n}\right) r T\left(b_{1}, b_{2}, \ldots, b_{n}\right)=0
$$

for all $r \in \mathcal{R}, a_{i}, b_{i} \in \mathcal{G}_{i}, i=1,2, \ldots, n$.

The following lemma is easily observed.

Lemma 2.2 ([9, Lemma 2.4]). Let $\mathcal{R}$ be a ring and $\Delta$ be a generalized Jordan triple higher derivation on $\mathcal{R}$. Then for all $x, y, z \in \mathcal{R}$ and for each $n \in \mathbb{N}_{0}$,

(a) $P_{n}(x, y, z)+P_{n}(z, y, x)=0$,

(b) $P_{n}(x, y, z)$ is additive in each variable.

Lemma 2.3 ([9, Lemma 2.5]). Let $\mathcal{R}$ be a semiprime ring and $\Delta$ be a generalized Jordan triple higher derivation on $\mathcal{R}$. If $n \in \mathbb{N}_{0}$ such that $P_{m}(x, y, z)=0$ for all $x, y, z \in \mathcal{R}$ and $m<n$, then

$$
P_{n}(x, y, z) r Q(x, y, z)=0
$$

for all $x, y, z, r \in \mathcal{R}$.

From Lemma 2.1 and Lemma 2.3 we immediately get:

Corollary 2.4. Let $\mathcal{R}$ be a semiprime ring and $\Delta$ be a generalized Jordan triple higher derivation on $\mathcal{R}$. If $n \in \mathbb{N}_{0}$ such that $P_{m}(x, y, z)=0$ for all $x, y, z \in \mathcal{R}$ and $m<n$, then

$$
P_{n}(x, y, z) r Q(u, v, w)=0
$$

for all $x, y, z, r, u, v, w \in \mathcal{R}$.

Next we will give a theorem which plays an important role in the proof of our main theorem. For its proof, let us recall some basic results with related to the classical quotient ring. From now $\mathcal{U}$ always denotes the left Utumi quotient ring of $\mathcal{R} . \mathcal{U}$ can be characterized as a ring satisfying the following properties:

(a) $\mathcal{R}$ is a subring of $\mathcal{U}$.

(b) For each $q \in \mathcal{U}$, there exists a dense left ideal $\mathcal{I}_{q}$ of $\mathcal{R}$ such that $\mathcal{I}_{q} q \subseteq \mathcal{R}$.

(c) If $q \in \mathcal{U}$ and $\mathcal{I} q=0$ for some dense left ideal $\mathcal{I}$ of $\mathcal{R}$, then $q=0$. 
(d) If $\phi: \mathcal{I} \rightarrow \mathcal{R}$ is a left $\mathcal{R}$-module map from a dense left ideal $\mathcal{I}$ of $\mathcal{R}$ into $\mathcal{R}$, then there exists an element $q \in \mathcal{U}$ such that $\phi(i)=i q$ for all $i \in \mathcal{I}$. Up to isomorphisms, $\mathcal{U}$ is uniquely determined by the above four properties. If $\mathcal{R}$ is a (semi-)prime ring, then $\mathcal{U}$ is also a (semi-)prime ring. $\mathcal{U}$ always has identity element $e$.

Theorem 2.5. Let $\mathcal{R}$ be a semiprime ring and $\Delta$ be a generalized Jordan triple higher derivation on $\mathcal{R}$. If $n \in \mathbb{N}_{0}$ such that $P_{m}(x, y, z)=0$ for all $x, y, z \in \mathcal{R}$ and $m<n$, then

for all $x, y, z \in \mathcal{R}$.

$$
P_{n}(x, y, z) \in \mathcal{C}_{\mathcal{R}}
$$

Proof. From Corollary 2.4 we have

$$
\begin{aligned}
& Q\left(P_{n}(x, y, z), u, v\right) r Q\left(P_{n}(x, y, z), u, v\right) \\
= & \left(P_{n}(x, y, z) u v-v u P_{n}(x, y, z)\right) r Q\left(P_{n}(x, y, z), u, v\right) \\
= & P_{n}(x, y, z) u v r Q\left(P_{n}(x, y, z), u, v\right)-v u P_{n}(x, y, z) r Q\left(P_{n}(x, y, z), u, v\right) \\
= & 0
\end{aligned}
$$

for all $x, y, z, r, u, v \in \mathcal{R}$. Thus $Q\left(P_{n}(x, y, z), u, v\right)=0$ follows by the semiprimeness of $\mathcal{R}$. That is $P_{n}(x, y, z) u v=v u P_{n}(x, y, z)$ for all $x, y, z, u$, $v \in \mathcal{R}$. Let us now fix the coefficients $Q(x, y, z)$ and regard $P_{n}(x, y, z) u v=$ $v u P_{n}(x, y, z)$ as a generalized polynomial with related to the two variables $u, v$. Since $\mathcal{R}$ and $\mathcal{U}$ satisfy the same generalized polynomial identities, $P_{n}(x, y, z) u v$ $=v u P_{n}(x, y, z)$ for all $u, v \in \mathcal{U}$. Taking $v=e$ in $P_{n}(x, y, z) u v=v u P_{n}(x, y, z)$ yields that $P_{n}(x, y, z) u=u P_{n}(x, y, z)$ for all $u \in \mathcal{U}$. In particular, $P_{n}(x, y, z) u$ $=u P_{n}(x, y, z)$ for all $u \in \mathcal{R}$. This shows that $P_{n}(x, y, z) \in \mathcal{C}_{\mathcal{R}}$. This completes the proof of this theorem. lary.

By Theorem 2.5 and Corollary 2.4 we immediately get the following corol-

Corollary 2.6. Let $\mathcal{R}$ be a semiprime ring and $\Delta$ be a generalized Jordan triple higher derivation on $\mathcal{R}$. If $n \in \mathbb{N}_{0}$ such that $P_{m}(x, y, z)=0$ for all $x, y, z \in \mathcal{R}$ and $m<n$, then

$$
P_{n}(x, y, z) Q(u, v, w)=0
$$

for all $x, y, z, u, v, w \in \mathcal{R}$. In particular, $P_{n}(x, y, z)[u, v]=0$ for all $x, y, z, u, v \in$ $\mathcal{R}$.

Now we are in a position to state our main theorem of this paper.

Theorem 2.7. Let $\mathcal{R}$ be a 2-torsion free semiprime ring. Then every generalized Jordan triple higher derivation on $\mathcal{R}$ is a generalized higher derivation.

Proof. We assume that $\Delta$ is a generalized Jordan triple higher derivation on $\mathcal{R}$ with the associated Jordan triple higher derivation $D$. Actually, by [5, Theorem 1.2], $D$ is a higher derivation on $\mathcal{R}$. Let us first prove that $P_{n}(x, y, z)=0$ 
for all $x, y, z \in \mathcal{R}$ and for each $n \in \mathbb{N}_{0}$ by induction. When $n=0$, it is easy to obtain $P_{0}(x, y, z)=0$ for all $x, y, z \in \mathcal{R}$. When $n=1$, by [13, Theorem 2.8] we know that $P_{1}(x, y, z)=0$ for all $x, y, z \in \mathcal{R}$. We now suppose that $P_{m}(x, y, z)=0$ for all $x, y, z \in \mathcal{R}$ and $m<n$. Then we have $P_{n}(x, y, z) r Q(u, v, w)=0$ for all $x, y, z, r, u, v, w \in \mathcal{R}$ by Corollary 2.4. The following proof is divided into two different cases.

Case 1. If $Q(u, v, w)=0$ is satisfied for all $u, v, w \in \mathcal{R}$, then $u v w=w v u$. Since $\mathcal{R}$ and $\mathcal{U}$ satisfy the same generalized polynomial identities, we have $u v w=w v u$ for all $u, v, w \in \mathcal{U}$. Taking $w=e$ into $u v w=w v u$ yields the commutativity of $\mathcal{U}$ and that of $\mathcal{R}$.

For any $x, y, z \in \mathcal{R}$, let us set $a=\delta_{n}\left(x y z^{3}+z^{3} y x\right)$. Then

$$
\begin{aligned}
a= & \delta_{n}\left(x y z^{3}+z^{3} y x\right) \\
= & \sum_{i+j+k=n} \delta_{i}(x) d_{j}(y) d_{k}\left(z^{3}\right)+\sum_{i+j+k=n} \delta_{i}\left(z^{3}\right) d_{j}(y) d_{k}(x) \\
= & \sum_{i+j+k=n} \delta_{i}(x) d_{j}(y)\left[\sum_{h+l+m=k} d_{h}(z) d_{l}(z) d_{m}(z)\right] \\
& +\sum_{i+j+k=n}\left[\sum_{\alpha+\beta+\gamma=i} \delta_{\alpha}(z) d_{\beta}(z) d_{\gamma}(z)\right] d_{j}(y) d_{k}(x) \\
= & \sum_{i+j+h+l+m=n} \delta_{i}(x) d_{j}(y) d_{h}(z) d_{l}(z) d_{m}(z) \\
& +\sum_{\alpha+\beta+\gamma+j+k=n} \delta_{\alpha}(z) d_{\beta}(z) d_{\gamma}(z) d_{j}(y) d_{k}(x) \\
= & \sum_{i+j+h+l+m=n, l+m \neq 0} \delta_{i}(x) d_{j}(y) d_{h}(z) d_{l}(z) d_{m}(z) \\
& +\sum_{i+j+h=n} \delta_{i}(x) d_{j}(y) d_{h}(z) z^{2} \\
& +\sum_{\alpha+\beta+\gamma+j+k=n} \delta_{\alpha}(z) d_{\beta}(z) d_{\gamma}(z) d_{j}(y) d_{k}(x) .
\end{aligned}
$$

On the other hand, by the commutativity of $\mathcal{R}$, we have

$$
\begin{aligned}
a & =\delta_{n}\left(x y z^{3}+z^{3} y x\right)=\delta_{n}((z y x) z z+z z(z y x)) \\
& =\sum_{i+j+k=n} \delta_{i}(z y x) d_{j}(z) d_{k}(z)+\sum_{i+j+k=n} \delta_{i}(z) d_{j}(z) d_{k}(z y x) \\
& =\sum_{i+j+k=n} \delta_{i}(x y z) d_{j}(z) d_{k}(z)+\sum_{i+j+k=n} \delta_{i}(z) d_{j}(z) d_{k}(z y x) \\
& =\sum_{i+j+k=n, j+k \neq 0}\left[\sum_{\zeta+\lambda+\nu=i} \delta_{\zeta}(x) d_{\lambda}(y) d_{\nu}(z)\right] d_{j}(z) d_{k}(z)+\delta_{n}(x y z) z^{2}
\end{aligned}
$$




$$
\begin{aligned}
& +\sum_{i+j+k=n} \delta_{i}(z) d_{j}(z)\left[\sum_{p+q+s=k} d_{p}(z) d_{q}(y) d_{s}(x)\right] \\
= & \sum_{\zeta+\lambda+\nu+j+k=n, j+k \neq 0} \delta_{\zeta}(x) d_{\lambda}(y) d_{\nu}(z) d_{j}(z) d_{k}(z)+\delta_{n}(x y z) z^{2} \\
& +\sum_{i+j+p+q+s=n} \delta_{i}(z) d_{j}(z) d_{p}(z) d_{q}(y) d_{s}(x) .
\end{aligned}
$$

Combining (2.1) with (2.2) yields

$$
\left[\delta_{n}(x y z)-\sum_{i+j+h=n} \delta_{i}(x) d_{j}(y) d_{h}(z)\right] z^{2}=0
$$

for all $x, y, z \in \mathcal{R}$. That is,

$$
P_{n}(x, y, z) z^{2}=0
$$

for all $x, y, z \in \mathcal{R}$. Thus we get

$$
P_{n}(x, y, z) z r P_{n}(x, y, z) z=P_{n}(x, y, z) z^{2} r P_{n}(x, y, z)=0
$$

for all $x, y, z, r \in \mathcal{R}$. By the semiprimeness of $\mathcal{R}$, we obtain

$$
P_{n}(x, y, z) z=0
$$

for all $x, y, z \in \mathcal{R}$. Linearization of (2.3) leads to

$$
P_{n}(x, y, z) s+P_{n}(x, y, s) z=0
$$

for all $x, y, z, s \in \mathcal{R}$. It follows from (2.3) and (2.4) that

$$
\begin{aligned}
P_{n}(x, y, z) s r P_{n}(x, y, z) s & =-P_{n}(x, y, s) z r P_{n}(x, y, z) s \\
& =-P_{n}(x, y, z) z r P_{n}(x, y, s) s=0 .
\end{aligned}
$$

Then $P_{n}(x, y, z) s=0$ for all $x, y, z, s \in \mathcal{R}$ and hence $P_{n}(x, y, z)=0$ for all $x, y, z \in \mathcal{R}$.

Case 2. If there exist $u, v, w \in \mathcal{R}$ such that $Q(u, v, w) \neq 0$, then by Corollary 2.6, it follows that $P_{n}(x, y, z) Q(u, v, w)=0$ for all $x, y, z \in \mathcal{R}$. Then for any positive integer $l$ and for arbitrarily chosen $x, y, z \in \mathcal{R}, \operatorname{Ann}\left(P_{n}(x, y, z)^{l}\right)$ is an ideal of $\mathcal{R}$ by Theorem 2.5. Furthermore, $\operatorname{Ann}\left(P_{n}(x, y, z)^{l}\right)$ is a semiprime ideal of $\mathcal{R}$. Indeed, for a given $t \in \mathcal{R}$, if $\operatorname{trt} \in \operatorname{Ann}\left(P_{n}(x, y, z)^{l}\right)$ for all $r \in \mathcal{R}$, then $(t r t) P_{n}(x, y, z)^{l}=0$. Thus $\left[t P_{n}(x, y, z)^{l}\right] r\left[t P_{n}(x, y, z)^{l}\right]=0$ and hence $t \in$ $\operatorname{Ann}\left(P_{n}(x, y, z)^{l}\right)$ by the semiprimeness of $\mathcal{R}$. Obviously, $\operatorname{Ann}\left(P_{n}(x, y, z)^{l}\right) \subseteq$ $\operatorname{Ann}\left(P_{n}(x, y, z)^{l+1}\right)$ for any positive integer $l$. It is easy to check that $\mathcal{I}=$ $\bigcup_{l \geq 1} \operatorname{Ann}\left(P_{n}(x, y, z)^{l}\right)$ is also a semiprime ideal of $\mathcal{R}$ and $\mathcal{R} / \mathcal{I}$ is a 2 -torsion free commutative semiprime ring by Corollary 2.6.

Now we assert that the additive maps $d_{i}(1 \leq i \leq n)$ in $D(1 \leq i \leq n)$ and the additive maps $\delta_{i}$ in $\Delta$ keep $\mathcal{I}$ invariant. To get this assertion we still use induction method.

Obviously, $d_{0}=i d_{\mathcal{R}}$ and $\delta_{0}=i d_{\mathcal{R}}$ both keep $\mathcal{I}$ invariant. 
When $i=1$. For any $q \in \mathcal{I}$, then $q \in \operatorname{Ann}\left(P_{n}(x, y, z)^{l}\right)$ for some positive integer $l$ and for the fixed $x, y, z \in \mathcal{R}$. So

$$
0=d_{1}\left(q P_{n}(x, y, z)^{l}\right)=d_{1}(q) P_{n}(x, y, z)^{l}+q d_{1}\left(P_{n}(x, y, z)^{l}\right)
$$

for the fixed $x, y, z \in \mathcal{R}$. Multiplication of (2.5) by $P_{n}(x, y, z)^{l}$ leads to

$$
d_{1}(q) P_{n}(x, y, z)^{2 l}=0
$$

for the chosen and fixed $x, y, z \in \mathcal{R}$. This shows that $d_{1}(q) \in \mathcal{I}$ and that $d_{1}$ keeps $\mathcal{I}$ invariant. As for $\delta_{1}$, we have

$$
\begin{aligned}
0= & \delta_{1}\left(q P_{n}(x, y, z)^{l} r+r P_{n}(x, y, z)^{l} q\right) \\
= & \delta_{1}(q) P_{n}(x, y, z)^{l} r+q d_{1}\left(P_{n}(x, y, z)^{l}\right) r+q P_{n}(x, y, z)^{l} d_{1}(r) \\
& +\delta_{1}(r) P_{n}(x, y, z)^{l} q+r d_{1}\left(P_{n}(x, y, z)^{l}\right) q+r P_{n}(x, y, z)^{l} d_{1}(q) \\
= & \delta_{1}(q) P_{n}(x, y, z)^{l} r+q d_{1}\left(P_{n}(x, y, z)^{l}\right) r
\end{aligned}
$$

for the fixed $x, y, z \in \mathcal{R}$ and for all $r \in \mathcal{R}$. By the fact that $\mathcal{R}$ and $\mathcal{U}$ satisfy the same generalized polynomial identities, we obtain $0=\delta_{1}(q) P_{n}(x, y, z)^{l} r+$ $q d_{1}\left(P_{n}(x, y, z)^{l}\right) r$ for all $r \in \mathcal{U}$. Taking $r=e$ in (2.7) yields

$$
0=\delta_{1}(q) P_{n}(x, y, z)^{l}+q d_{1}\left(P_{n}(x, y, z)^{l}\right) .
$$

Multiplication of $(2.8)$ by $P_{n}(x, y, z)^{l}$ gives

$$
\delta_{1}(q) P_{n}(x, y, z)^{2 l}=0
$$

for the chosen and fixed $x, y, z \in \mathcal{R}$. This shows that $\delta_{1}(q) \in \mathcal{I}$ and that $\delta_{1}$ keeps $\mathcal{I}$ invariant.

We assume $d_{m}(m<n)$ and $\delta_{m}(m<n)$ keep $\mathcal{I}$ invariant. Let us see the case of $d_{n}$ and $\delta_{n}$, respectively. For any $q \in \mathcal{I}$, then $q \in \operatorname{Ann}\left(P_{n}(x, y, z)^{l}\right)$ for some positive integer $l$ and for the fixed $x, y, z \in \mathcal{R}$. Thus

$$
\begin{aligned}
0=d_{n}\left(q P_{n}(x, y, z)^{l}\right)= & d_{n}(q) P_{n}(x, y, z)^{l}+q d_{n}\left(P_{n}(x, y, z)^{l}\right) \\
& +\sum_{i+j=n ; i, j<n} d_{i}(q) d_{j}\left(P_{n}(x, y, z)^{l}\right) .
\end{aligned}
$$

According to the assumption, $d_{i}(q) \in \mathcal{I}$ for all $i<n$. Multiplication of $(2.9)$ by $P_{n}(x, y, z)^{l}$ gives

$$
0=d_{n}(q) P_{n}(x, y, z)^{2 l}
$$


for the chosen and fixed $x, y, z \in \mathcal{R}$. So $d_{n}(q) \in \mathcal{I}$ and $d_{n}$ keeps $\mathcal{I}$ invariant. As for $\delta_{n}$, we have

$$
\begin{aligned}
0= & \delta_{n}\left(q P_{n}(x, y, z)^{l} r+r P_{n}(x, y, z)^{l} q\right) \\
= & \delta_{n}(q) P_{n}(x, y, z)^{l} r+r P_{n}(x, y, z)^{l} d_{n}(q) \\
& +\sum_{i+j+k=n: i \neq n} \delta_{i}(q) d_{j}\left(P_{n}(x, y, z)^{l}\right) d_{k}(r) \\
& +\sum_{i+j+k=n, k \neq n} \delta_{i}(r) d_{j}\left(P_{n}(x, y, z)^{l}\right) d_{k}(q)
\end{aligned}
$$

for the chosen and fixed $x, y, z \in \mathcal{R}$ and for all $r \in \mathcal{R}$. In view of the assumptions, $\delta_{i}(q) \in \mathcal{I}$ for all $i<n$ and $d_{k}(q) \in \mathcal{I}$ for all $k<n$. Multiplication of (2.11) by $P_{n}(x, y, z)^{l}$ gives

$$
0=\delta_{n}(q) P_{n}(x, y, z)^{2 l} r
$$

for the chosen and fixed $x, y, z \in \mathcal{R}$ and for all $r \in \mathcal{R}$. Again, by the fact that $\mathcal{R}$ and $\mathcal{U}$ satisfy the same generalized polynomial identities, we obtain $0=\delta_{n}(q) P_{n}(x, y, z)^{2 l} r$ for all $r \in \mathcal{U}$. Substituting $r=e$ into (2.12) yields

$$
0=\delta_{n}(q) P_{n}(x, y, z)^{2 l} .
$$

Thus $\delta_{n}(q) \in \mathcal{I}$ and $\delta_{n}$ keeps $\mathcal{I}$ invariant.

Therefore the additive maps $d_{i}(0 \leq i \leq n)$ in $D$ and the additive maps $\delta_{i}(0 \leq i \leq n)$ in $\Delta$ both can be induced to $\mathcal{R} / \mathcal{I}$ as follows

$$
\overline{\delta_{i}}(\bar{r})=\delta_{i}(r)+\mathcal{I}, \quad \overline{d_{i}}(\bar{r})=d_{i}(r)+\mathcal{I}
$$

for all $\bar{r} \in \mathcal{R} / \mathcal{I}$. It is easy to check that $\bar{\Delta}$ is a generalized Jordan triple higher derivation on $\mathcal{R} / \mathcal{I}$ with an associated Jordan triple higher derivation $\bar{D}$. Since $\mathcal{R} / \mathcal{I}$ is a 2 -torsion free commutative semiprime ring, we immediately get $\overline{P_{n}}(\bar{r}, \bar{s}, \bar{t})=0$ for all $\bar{r}, \bar{s}, \bar{t} \in \mathcal{R} / \mathcal{I}$ by the proof of Case 1 . This implies that $P_{n}(r, s, t) \in \mathcal{I}$ for all $r, s, t \in \mathcal{R}$. In particular, there exists a positive integer $L$ such that $P_{n}(x, y, z) \in \operatorname{Ann}\left(P_{n}(x, y, z)^{L}\right)$ for the fixed $x, y, z \in \mathcal{R}$. Therefore $P_{n}(x, y, z)^{L+1}=0$. However, there is no nonzero nilpotent element in the center of a semiprime ring. Since $x, y, z$ are arbitrarily chosen in $\mathcal{R}$ at the beginning of this step, we conclude that $P_{n}(x, y, z)=0$ for all $x, y, z \in \mathcal{R}$.

From Case 1 and Case 2 it follows that $P_{n}(x, y, z)=0$ for all $x, y, z \in \mathcal{R}$ and for each $n \in \mathbb{N}_{0}$. It remains to prove that $\Delta=\left(\delta_{i}\right)_{i \in \mathbb{N}_{0}}$ is a generalized higher derivation on $\mathcal{R}$.

Let us set $A_{n}(x, y)=\delta_{n}(x y)-\sum_{i+j=n} \delta_{i}(x) d_{j}(y)$ for all $x, y \in \mathcal{R}$ and for each $n \in \mathbb{N}_{0}$. It is easy to see that $A_{n}(x, y)$ is additive in each variable. When $n=0$, obviously, $A_{0}(x, y)=0$. When $n=1$, by [13, Theorem 2.8], it follows that $A_{1}(x, y)=0$. Using induction method, we now assume that $A_{m}(x, y)=0$ for all $x, y \in \mathcal{R}$ and $m<n$. Let us write $B=\delta_{n}(x y r x y)$ for all $x, y, r \in \mathcal{R}$. 
Then we have

(2.13)

$$
\begin{aligned}
B & =\delta_{n}(x(y r x) y)=\sum_{i+j+k=n} \delta_{i}(x) d_{j}(y r x) d_{k}(y) \\
& =\sum_{i+j+k=n} \delta_{i}(x)\left[\sum_{p+q+s=j} d_{p}(y) d_{q}(r) d_{s}(x)\right] d_{k}(y) \\
& =\sum_{i+p+q+s+k=n} \delta_{i}(x) d_{p}(y) d_{q}(r) d_{s}(x) d_{k}(y) \\
& =\sum_{i+p+q+s+k=n, q+s+k \neq 0} \delta_{i}(x) d_{p}(y) d_{q}(r) d_{s}(x) d_{k}(y)+\sum_{i+p=n} \delta_{i}(x) d_{p}(y) r x y .
\end{aligned}
$$

On the other hand, by the induction assumption we get

$$
\begin{aligned}
B & =\delta_{n}((x y) r(x y))=\sum_{i+j+k=n} \delta_{i}(x y) d_{j}(r) d_{k}(x y) \\
& =\sum_{i+j+k=n} \delta_{i}(x y) d_{j}(r)\left[\sum_{l+h=k} d_{l}(x) d_{h}(y)\right] \\
& =\sum_{i+j+l+h=n} \delta_{i}(x y) d_{j}(r) d_{l}(x) d_{h}(y) \\
& =\sum_{i+j+l+h=n, j+l+h \neq 0} \delta_{i}(x y) d_{j}(r) d_{l}(x) d_{h}(y)+\delta_{n}(x y) r x y \\
& =\sum_{i+j+l+h=n, j+l+h \neq 0}\left[\sum_{u+v=i} \delta_{u}(x) d_{v}(y)\right] d_{j}(r) d_{l}(x) d_{h}(y)+\delta_{n}(x y) r x y \\
& =\sum_{u+v+j+l+h=n, j+l+h \neq 0} \delta_{u}(x) d_{v}(y) d_{j}(r) d_{l}(x) d_{h}(y)+\delta_{n}(x y) r x y .
\end{aligned}
$$

Combining (2.13) with (2.14) we obtain

$$
\left[\delta_{n}(x y)-\sum_{i+p=n} \delta_{i}(x) d_{p}(y)\right] r x y=0
$$

for all $x, y, r \in \mathcal{R}$. That is

$$
A_{n}(x, y) r x y=0
$$

for all $x, y, r \in \mathcal{R}$. The linearization of (2.15) yields

$$
A_{n}(x, y) r z y=-A_{n}(z, y) r x y .
$$

By $(2.15),(2.16)$ and $[3$, Lemma 1.1] we have

$$
\left[A_{n}(x, y) r z y\right] s\left[A_{n}(x, y) r z y\right]=-A_{n}(x, y) r\left[z y s A_{n}(z, y)\right] r x y=0
$$


for all $x, y, z, r, s \in \mathcal{R}$. Since $\mathcal{R}$ is semiprime, $A_{n}(x, y) r z y=0$ for all $x, y, z, r \in$ $\mathcal{R}$. Using the same linearizing approach we can get $A_{n}(x, y) r z w=0$ for all $x, y, z, r, w \in \mathcal{R}$. In particular, $A_{n}(x, y) z r A_{n}(x, y) z=0$ and hence $A_{n}(x, y) z=$ 0 , which implies that $A_{n}(x, y)=0$. So $\Delta=\left(\delta_{i}\right)_{i \in \mathbb{N}_{0}}$ is a generalized higher derivation on $\mathcal{R}$ with an associated higher derivation $D=\left(d_{i}\right)_{i \in \mathbb{N}_{0}}$. This completes the proof of this theorem.

Another interesting question is that: under what conditions, a generalized Jordan higher derivation on $\mathcal{R}$ is a generalized Jordan triple higher derivation? In [5] Ferrero and Haetinger proved the following results.

Theorem 2.8 ([5, Theorem 1.3]). Let $R$ be a 2-torsion free ring and $\mathcal{L}$ be a Lie ideal of $\mathcal{R}$. Then every Jordan higher derivation of $\mathcal{R}$ (resp. of $\mathcal{L}$ into $\mathcal{R}$ ) is a Jordan triple higher derivation of $\mathcal{R}$ (resp. of $\mathcal{L}$ into $\mathcal{R})$.

Corollary 2.9 ([5, Corollary 1.4]). Let $\mathcal{R}$ be a 2-torsion free semiprime ring (resp. prime ring of characteristic different from 2 and $\mathcal{L}$ be a non-central Lie ideal of $\mathcal{R}$ ). Then every Jordan higher derivation on $\mathcal{R}$ (resp. of $\mathcal{L}$ into $\mathcal{R}$ ) is a higher derivation of $\mathcal{R}$ (resp. of $\mathcal{L}$ into $\mathcal{R})$.

Now we answer the above question under certain mild assumptions. Actually, the following theorem and corollary are common generalizations of Theorem 2.8 and Corollary 2.9, respectively.

Theorem 2.10. Let $\mathcal{R}$ be a 2-torsion free ring and $\mathcal{L}$ be a Lie ideal of $\mathcal{R}$. Then every generalized Jordan higher derivation of $\mathcal{R}$ (resp. of $\mathcal{L}$ into $\mathcal{R}$ ) is a generalized Jordan triple higher derivation of $\mathcal{R}$ (resp. of $\mathcal{L}$ into $\mathcal{R})$.

Proof. Suppose that $\mathcal{R}$ is a 2-torsion free ring and $\Delta$ is a generalized Jordan higher derivation on $\mathcal{R}$ (resp. of $\mathcal{L}$ into $\mathcal{R}$ ) with an associated Jordan higher derivation $D$. Then $D$ is also a Jordan triple higher derivation on $\mathcal{R}$ by Theorem 2.8. Now we claim that

$$
\delta_{n}(x y+y x)=\sum_{i+j=n} \delta_{i}(x) d_{j}(y)+\delta_{i}(y) d_{j}(x)
$$

for all $x, y \in \mathcal{R}$ (resp. $x, y \in \mathcal{L}$ ) and for each $n \in \mathbb{N}_{0}$. By the definition of generalized Jordan higher derivation, we have $\delta_{n}\left(x^{2}\right)=\sum_{i+j=n} \delta_{i}(x) d_{j}(x)$ for all $x \in \mathcal{R}$ and for each $n \in \mathbb{N}_{0}$. Then the above claim follows from the relations

$$
\begin{aligned}
\delta_{n}\left((x+y)^{2}\right) & =\sum_{l+m=n} \delta_{l}(x+y) d_{m}(x+y) \\
& =\sum_{l+m=n} \delta_{l}(x) d_{m}(x)+\delta_{l}(x) d_{m}(y)+\delta_{l}(y) d_{m}(x)+\delta_{l}(y) d_{m}(y)
\end{aligned}
$$

and

$$
\begin{aligned}
\delta_{n}\left((x+y)^{2}\right) & =\delta_{n}\left(x^{2}+x y+y x+y^{2}\right) \\
& =\delta_{n}\left(x^{2}\right)+\delta_{n}(x y+y x)+\delta_{n}\left(y^{2}\right)
\end{aligned}
$$




$$
=\delta_{n}(x y+y x)+\sum_{p+q=n} \delta_{p}(x) d_{q}(x)+\sum_{u+v=n} \delta_{u}(y) d_{v}(y) .
$$

Let us set $w=(x(x y+y x)+(x y+y x) x)$. By (2.17) and Corollary 2.9 we get

$$
\begin{aligned}
\delta_{n}(w)= & \sum_{i+j=n} \delta_{i}(x) d_{j}(x y+y x)+\sum_{i+j=n} \delta_{i}(x y+y x) d_{j}(x) \\
= & \sum_{i+j=n} \sum_{g+h=j} \delta_{i}(x) d_{g}(x) d_{h}(y)+2 \sum_{i+j+k=n} \delta_{i}(x) d_{j}(y) d_{k}(x) \\
& +\sum_{i+j=n} \sum_{k+l=i} \delta_{k}(y) d_{l}(x) d_{j}(x) .
\end{aligned}
$$

On the other hand,

$$
\begin{aligned}
\delta_{n}(w) & =\delta_{n}\left(\left(x^{2} y+y x^{2}\right)+2 x y x\right)=\delta_{n}\left(x^{2} y+y x^{2}\right)+2 \delta_{n}(x y x) \\
& =2 \delta_{n}(x y x)+\sum_{i+j=n} \sum_{g+h=i} \delta_{g}(x) d_{h}(x) d_{j}(y)+\sum_{i+j=n} \sum_{k+l=j} \delta_{i}(y) d_{k}(x) d_{l}(x) .
\end{aligned}
$$

Combining (2.18) with (2.19) we obtain

$$
\delta_{n}(x y x)=\sum_{i+j+k=n} \delta_{i}(x) d_{j}(y) d_{k}(x)
$$

for all $x, y \in \mathcal{R}$ and for each $n \in \mathbb{N}_{0}$. This shows that $\Delta$ is a generalized Jordan triple higher derivation with an associated Jordan triple higher derivation $D$. The proof of this theorem is completed.

In view of Theorem 2.7 and Theorem 2.10 we have:

Corollary 2.11. Let $\mathcal{R}$ be a semiprime ring of characteristic different from 2 (resp. prime ring of characteristic different from 2 and $\mathcal{L}$ be a non-central Lie ideal of $\mathcal{R})$. Then every generalized Jordan higher derivation on $\mathcal{R}$ (resp. of $\mathcal{L}$ into $\mathcal{R})$ is a generalized higher derivation of $\mathcal{R}$ (resp. of $\mathcal{L}$ into $\mathcal{R})$.

One can ask whether the Corollary 2.9 remains valid for Lie ideals of semiprime rings. Without any additional assumption this is not true in general. A counterexample was given in [5].

Acknowledgements. The authors would like to express sincere gratitude to the referee for his or her careful reading and making several corrections.

\section{References}

[1] N. Argac and E. Albas, On generalized $(\sigma, \tau)$-derivations, Sibirsk. Mat. Zh. 43 (2002), no. 6, 1211-1221; translation in Siberian Math. J. 43 (2002), no. 6, 977-984.

[2] M. Brešar, Jordan derivations on semiprime rings, Proc. Amer. Math. Soc. 104 (1988), no. 4, 1003-1006.

[3] _ Jordan mappings of semiprime rings, J. Algebra 127 (1989), no. 1, 218-228.

[4] J. M. Cusack, Jordan derivations on rings, Proc. Amer. Math. Soc. 53 (1975), no. 2, $321-324$. 
[5] M. Ferrero and C. Haetinger, Higher derivations and a theorem by Herstein, Quaest. Math. 25 (2002), no. 2, 249-257.

[6] I. N. Herstein, Jordan derivations of prime rings, Proc. Amer. Math. Soc. 8 (1957), 1104-1110.

[7] B. Hvala, Generalized derivations in rings, Comm. Algebra 26 (1998), no. 4, 1147-1166.

[8] W. Jing and S.-J. Lu, Generalized Jordan derivations on prime rings and standard operator algebras, Taiwanese J. Math. 7 (2003), no. 4, 605-613.

[9] Y.-S. Jung, Generalized Jordan triple higher derivations on prime rings, Indian J. Pure Appl. Math. 36 (2005), no. 9, 513-524.

[10] Y.-S. Jung and K.-H. Park, On generalized $(\alpha, \beta)$-derivations and commutativity in prime rings, Bull. Korean Math. Soc. 43 (2006), no. 1, 101-106.

[11] _ On prime and semiprime rings with permuting 3-derivations, Bull. Korean Math. Soc. 44 (2007), no. 4, 789-794.

[12] F. Wei, *-generalized differential identities of semiprime rings with involution, Houston J. Math. 32 (2006), no. 3, 665-681.

[13] F. Wei and Z.-K. Xiao, Generalized Jordan derivations and its pairs on semiprime rings, Demonstratio Math., in press.

FENG WEI

Department of Mathematics

Beijing Institute of Technology

BeiJing 100081, China

E-mail address: daoshuo@bit.edu.cn

ZHANKUI XiAO

Department of Mathematics

Beijing Institute of Technology

BeIJing 100081, China

E-mail address: zhkxiao@bit.edu.cn 